\title{
Suspected appendicitis and COVID-19, a change in investigation and management-a multicentre cohort study
}

\author{
W. English ${ }^{1,2}$ (D) N. Habib Bedwani ${ }^{1}$ (D) $\cdot$ C. Smith ${ }^{1}$ (D) E. Doganay ${ }^{3} \cdot$ M. Marsden ${ }^{3} \cdot$ S. Muse $^{4} \cdot$ W. K. Mak ${ }^{4} \cdot$ M. Chana $^{4}$. \\ J. Eves ${ }^{5} \cdot$ V. Shatkar ${ }^{1}$
}

Received: 14 September 2020 / Accepted: 2 November 2020 / Published online: 9 November 2020

(C) Springer-Verlag GmbH Germany, part of Springer Nature 2020

\begin{abstract}
Purpose The COVID-19 pandemic has reformed global healthcare delivery. On 25 March 2020, Intercollegiate guidelines were published in the UK to promote safe surgical provision during the COVID-19 outbreak advocating non-operative management or avoidance of laparoscopy when surgery is essential. The effects of this on the investigation and management of appendicitis remain unknown.
\end{abstract}

Methods We performed a multicentre, prospective, observational study from the start of the new guidelines to the 6th of May 2020. We included all patients referred to surgical teams with suspected appendicitis. A recent historical cohort was identified for comparison. The primary outcome was the impact of the COVID-19 pandemic on the use of non-operative management in appendicitis. Secondary outcomes included imaging, negative appendicectomy rate (NAR), length of stay (LOS) and 30-day complications.

Results A total of 63/164 (38\%) patients compared to 79/191 (41\%) were diagnosed with appendicitis before and after the guidelines were introduced $(p=0.589)$. CT scanning increased $(71 / 164$ vs $105 / 191 ; p=0.033)$ while ultrasound scanning decreased $(71 / 164$ vs $62 / 191 ; p=0.037)$. Appendicitis was more likely to be managed non-operatively $(11 / 63$ vs $51 / 79$; $p<0.001)$ and, of those managed surgically, with an open approach $(3 / 52$ vs $26 / 28 p<0.001)$. The NAR also reduced $(5 / 52$ vs $0 / 28 ; p=0.157$ ). LOS was shorter in non-operatively managed patients ( 1 day vs 3 days; $p<0.001)$ without a difference in complications $(10 / 51$ vs $4 / 28 ; p=0.760)$.

Conclusion Introduction of the guidelines was associated with changes in practice. Despite these changes, short-term complications did not increase and LOS decreased. Questions remain on the longer-term complication rates in non-operatively managed patients.

Keywords COVID-19 · Appendicitis · Laparoscopy $\cdot$ RIF pain

W. English and N. Habib Bedwani contributed equally to all aspects of the work and are joint first authors.

Preliminary data (single centre) from this study was submitted to the British Journal of Surgery as a "special letter to the editor" and accepted for publication (19th May 2020 - Investigation and management of suspected appendicitis during the COVID-19 pandemic DOI:https://doi. org/10.1002/bjs.11787).

\section{W. English}

Billy.english100@gmail.com

1 Department of General Surgery, Queens Hospital, BHR NHS Trust, London, UK

2 National Bowel Research Centre, Blizard Institute, QMUL, London, UK
Department of General Surgery, Royal London Hospital, Barts Health NHS Trust, London, UK

4 Department of General Surgery, The Royal Devon and Exeter Hospital NHS Foundation Trust, Exeter, UK

5 Department of General Surgery, St Mary's Hospital, Imperial College Healthcare NHS Trust, London, UK 


\section{Introduction}

The COVID-19 (Coronavirus Disease 2019) pandemic has impacted surgical provision worldwide $[1,2]$. Perioperative SARS-CoV-2 (severe acute respiratory distress coronavirus 2) infection is associated with poor outcomes with increased risks of pulmonary complications contributing to a 30 -day mortality of $23.1 \%$ for gastrointestinal surgery [3]. At the time of publication, the UK has reported over 300,000 laboratory confirmed COVID19 cases and 40,000 deaths [4]. Due to the UK's evolving COVID-19 pandemic, initial intercollegiate general surgery guidance was released on 26 March 2020 recommending significant changes to practice to all patients in all UK hospitals [5]. Given the increased risks associated with surgical intervention during the pandemic, non-operative management has been encouraged where possible [5]. Where non-operative management is inappropriate, the guidance recommends avoiding laparoscopy [6] because of the potential risk of aerosolisation [7]. Additionally all patients referred acutely or requiring emergency surgery should be investigated for concurrent COVID-19 infection [5].

Throughout the pandemic, acutely unwell patients continue to present to general surgical teams. Acute appendicitis is one of the commonest general surgical emergencies, with an estimated lifetime risk of $7-8 \%$ [8]. Under normal circumstances, the standard management would be laparoscopic appendicectomy [9]. A laparoscopic compared with an open approach is preferred as it is associated with a lower incidence of wound infection, shorter hospital stays and a faster return to normal activities [10].

Non-operative management of appendicitis with antibiotic therapy is an alternative option that can be safely used in certain cases with close monitoring [11], although this remains controversial with concerns over the risk of recurrence [12]. At 5 years, $39 \%$ of patients initially managed nonoperatively will have an appendicectomy due to recurrence or complication [13].

In an attempt to avoid the risks of surgical intervention during the COVID-19 pandemic [6], non-operative management with antibiotics has been suggested as a treatment option $[14,15]$. Preliminary data from our single centre was encouraging, with those managed non-operatively having shorter length of stay (LOS) [15].

The effect of the early phase of the COVID-19 pandemic in the UK on the management and short-term outcomes of adult patients with suspected appendicitis is unknown. The primary aim of this observational study is to determine if the COVID19 pandemic increases the use of non-operative management in appendicitis. The secondary aims include determining if there is a difference in LOS, use of CT imaging, the negative appendicectomy rate (NAR), the complication rate and the readmission rate in both cohorts.

\section{Methods}

This multicentre prospective observational study included adult patients who presented to hospital between 26 March and 6 May 2020 with suspected appendicitis (cohort B). Patients were included from four NHS Trusts (Appendix A). Adult patients ( $>17$ years) were included if appendicitis was in the differential diagnosis on referral from emergency departments or primary care. Eligible patients were identified through analysis of daily admission records.

A historical cohort of patients was chosen from 1 March to 25 March 2020 as a comparison group (cohort A) immediately prior to the publication of the Intercollegiate General Surgery Guidance on COVID-19 on 26 March 2020 [5] for direct comparison. Patients were identified through scrutiny of the daily admission records. Management decisions were clinician-led following discussion and agreement with patients.

\section{Data collection}

Demographic, clinical, pathological, radiological and histological data were collected from patient notes and the electronic health record (EHR). Age, gender, ethnicity, clinical diagnosis, management plan, operative findings, LOS (to include readmission), complications and follow-up were recorded. The EHR was used to collect routine laboratory blood tests, polymerase chain reaction (PCR) swab tests for SARS$\mathrm{CoV}-2$, radiological results; computed tomography (CT) of the chest, abdomen and pelvis and ultrasound scans (USS) of the abdomen and pelvis and histology results. Other data collected included time to imaging, time to surgery and follow-up including readmission and development of complications. Patients were followed up for 30 days post admission date for further admissions, development of complications and clinic follow-up.

\section{Diagnosis of appendicitis}

In all patients, the diagnostic methods used to establish the diagnosis (e.g. imaging (USS/CT), clinically suspected) were recorded. In patients undergoing an operation, the final diagnosis of appendicitis was made through evaluation of histological data for evidence of acute appendicitis or appendicular neoplasm. 


\section{Study approval}

The UK National Research Ethics Service decision tool (http://www.hra-decisiontools.org.uk) confirmed this study does not require ethical approval. It was registered within each trust as a service evaluation.

\section{Statistical analysis}

Variables of interest were assessed for normality using the D'Agostino-Pearson test. Categorical variables are expressed as frequency and were analysed using Fischer's exact test. Continuous variables are expressed as medians or means with 95\% confidence intervals and were analysed using the Student's $t$ test or Mann-Whitney $U$ test (as appropriate). Tests were performed using GraphPad Prism 8 (GraphPad Software, San Diego, California, USA) and a $p$ value of $<$ 0.05 was considered significant.

\section{Results}

\section{Patients}

Within 6 weeks of the new Intercollegiate guidelines, 191 patients met the inclusion criteria, of which 79/191 (41\%) had appendicitis. The rate of appendicitis was not statistically different from cohort A 63/164 (38\%), $(p=0.589)$. There were no significant differences in age or gender between the two cohorts, or a change in the number of non-appendicitis diagnoses (Table 1). In patients who were treated for appendicitis, there were no significant differences in baseline demographics. There was no demonstrable difference in white cell count (WCC) $\left(12.6\right.$ vs $\left.13.2 \times 10^{9} / \mathrm{L} ; p=0.480\right)$, neutrophil count $\left(10.5\right.$ vs $\left.10.9 \times 10^{9} / \mathrm{L} ; p=0.530\right)$ or C-reactive protein (CRP) (39 vs $23 \mathrm{mg} / \mathrm{L} ; p=0.304$ ) on admission in patients diagnosed with appendicitis between cohorts (Table 2).

\section{Imaging}

After the introduction of the guidelines, significantly more patients with possible appendicitis underwent $\mathrm{CT}$ scanning of the abdomen and pelvis $(105 / 191$ vs $71 / 164 ; p=0.033)$ and thorax $(66 / 191$ vs $9 / 164 ; p<0.001)$ (Fig. 1). Concurrently, there was a significant decrease in ultrasound examinations $(62 / 191$ vs $71 / 164 ; p=0.037)$. There was no difference in CT-reported complications of appendicitis between the cohorts $(2 / 32$ vs $8 / 48 ; p=0.301)$.

\section{Operative management}

Significantly fewer patients underwent surgery following introduction of the guidelines $(52 / 63$ vs $28 / 79 ; p<0.001)$
(Fig. 2a). When surgery was undertaken, laparoscopy was rarely used $(49 / 52$ vs $2 / 28 ; p<0.001)$ (Fig. $2 b)$.

\section{Non-operative management}

There was a statistically significant increase in the number of patients managed non-operatively between cohorts (11/63 vs $51 / 79 ; p<0.001)$. Within cohort $\mathrm{B}$, patients who were managed non-operatively had lower admission inflammatory markers (WCC 12.2 vs $15.2 \times 10^{9} / \mathrm{L} ; p=0.004$; neutrophil count 9.74 vs $12.8 \times 10^{9} / \mathrm{L} ; p=0.002$; CRP 18 vs $39 \mathrm{mg} / \mathrm{L}$; $p=0.045$ ) (Fig. 3).

\section{Negative appendicectomy rate}

There were no negative appendicectomies following the introduction of the guidelines compared to $10 \%$ prior to the guidelines. The difference was not statistically significant $(0 / 28$ vs $5 / 52 ; p=0.157)$.

\section{Length of stay}

The LOS was significantly shorter in patients undergoing nonoperative compared to operative management following introduction of the guidelines ( 1 day vs 3 days; $p<0.001$ ) (Fig. 4). LOS was also shorter for all patients being treated for appendicitis after introduction of the guidelines ( 2.5 days vs 2 days; $p=0.003$ ).

\section{Follow-up}

Following introduction of the guidelines, more patients were followed up in surgical clinics $(16 / 164$ vs $58 / 191 ; p<0.001)$ although without a difference in follow-up duration (2.5 days vs 5 days; $p=0.051$ ). A similar distribution was seen in those diagnosed with appendicitis in the two cohorts ( $4 / 63$ vs $34 / 79$; $p<0.001$; and 7 days vs 10 days $p=0.541$ ). Within cohort $\mathrm{B}$, patients were more likely to be followed up if they had nonoperative rather than operative management (30/51 vs $4 / 28$; $p=0.002$ ) although without a significant difference in followup duration (12 days vs 8 days; $p=0.092$ ).

\section{Complications and readmission}

Patients with any severity complications (Clavien-Dindo [16]) across the two cohorts were comparable (11/63 vs $14 / 79$; $p>0.999$ ). We did not demonstrate any difference in complication rate between patients within cohort $\mathrm{B}$ who were managed non-operatively versus operatively ( $4 / 28$ vs $10 / 51 ; p=$ 0.760 ). Of the 10 patients managed non-operatively with complications, six patients managed non-operatively following introduction of the guidelines required readmission for further antibiotics, and two were initially treated with radiological 
Table 1 Key demographic and process measures for all patients in cohorts $\mathrm{A}$ and $\mathrm{B}$, before (1 March-25 March 2020) and after (26 March-6 April 2020) implementation of new Intercollegiate guidance respectively

\begin{tabular}{llllll}
\hline & & Cohort A $(n=164)$ & Cohort B $(n=191)$ & $p$ & CI \\
\hline Age (years) & & $34(17-85)^{\dagger}$ & $33(17-93)^{\dagger}$ & 0.547 & $-4.621-1.930$ \\
Gender & Male & 59 & 77 & 0.444 & N/A \\
& Female & 105 & 114 & 0.444 & N/A \\
Diagnosis & Appendicitis & 63 & 79 & 0.589 & N/A \\
& Gynaecological & 21 & 14 & 0.108 & N/A \\
& Urinary tract & 13 & 9 & 0.270 & N/A \\
& NSAP & 44 & 55 & 0.723 & N/A \\
& Other & 23 & 34 & 0.385 & N/A \\
Imaging & CT thorax & 5 & 64 & $<0.0001^{*}$ & N/A \\
& CT abdomen & 71 & 105 & $0.033^{*}$ & N/A \\
& USS & 71 & 62 & $0.037^{*}$ & N/A \\
Cov. PCR & & 9 & 71 & $<0.0001^{*}$ & N/A \\
Followed up & & 16 & 58 & $<0.0001^{*}$ & N/A \\
LOF (days) & & $2.5(1-17)^{\dagger}$ & $5(1-30)^{\dagger}$ & 0.051 & $-0.381-7.916$ \\
LOS (days) & & $1(0-31)^{\dagger}$ & $1(0-16)^{\dagger}$ & $0.044^{*}$ & -0.847-0.332 \\
\hline
\end{tabular}

$C T$, computed tomography; $L O F$, length of follow-up; $L O S$, length of stay; N/A, not applicable; USS, ultrasound scan; $C I, 95 \%$ confidence interval; Cov. PCR, SARS-CoV-2 polymerase chain reaction performed

*Values showing statistical significance

${ }^{\dagger}$ Values demonstrate medians and range

Table 2 Key demographic, process and outcome measures for patients diagnosed with appendicitis in cohorts A and B, before (1 March-25 March 2020) and after (26 March-6 April 2020) implementation of new Intercollegiate guidance respectively

\begin{tabular}{|c|c|c|c|c|c|}
\hline & & Cohort A $(n=63)$ & Cohort B $(n=79)$ & $p$ & $\mathrm{CI}$ \\
\hline Age (years) & & $38(17-81)^{\dagger}$ & $33(17-93)^{\dagger}$ & 0.165 & $-8.557-1.644$ \\
\hline \multirow[t]{2}{*}{ Gender } & Male & 30 & 48 & 0.125 & N/A \\
\hline & Female & 33 & 30 & 0.125 & N/A \\
\hline \multirow[t]{3}{*}{ Imaging } & CT thorax & 4 & 28 & $<0.0001 *$ & N/A \\
\hline & CT abdomen & 32 & 48 & 0.307 & N/A \\
\hline & USS & 23 & 21 & 0.273 & N/A \\
\hline \multirow[t]{3}{*}{ Admission laboratory results } & WCC & $12.6(3.6-24.3)^{\dagger}$ & $13.2(3.7-20.1)^{\dagger}$ & 0.480 & $-1.194-1.883$ \\
\hline & Neutrophils & $10.5^{\wedge}$ & $10.9^{\wedge}$ & 0.530 & $-1.000-1.935$ \\
\hline & CRP & $39(1-373)^{\dagger}$ & $23(1-356)^{\dagger}$ & 0.304 & $-39.07-13.01$ \\
\hline \multirow[t]{2}{*}{ Management } & Conservative & 11 & 51 & $<0.0001^{*}$ & N/A \\
\hline & Surgical & 52 & 28 & $<0.0001^{*}$ & N/A \\
\hline \multirow[t]{2}{*}{ Surgical Mx } & Laparoscopic & 49 & 2 & $<0.0001^{*}$ & N/A \\
\hline & Open & 3 & 26 & $<0.0001^{*}$ & N/A \\
\hline Cov. PCR & & 9 & 28 & $0.006^{*}$ & N/A \\
\hline NAR & & 5 & 0 & 0.157 & N/A \\
\hline Followed up & & 4 & 34 & $<0.0001 *$ & N/A \\
\hline LOF (days) & & $7.25^{\wedge}$ & $9.67^{\wedge}$ & 0.541 & $-5.528-10.36$ \\
\hline LOS (days) & & $2.5(0-9)^{\dagger}$ & $2(0-14)^{\dagger}$ & $0.003 *$ & $-1.468-0.0271$ \\
\hline
\end{tabular}

CI, 95\% confidence interval; Cov. $P C R$, severe acute respiratory syndrome coronavirus 2 polymerase chain reaction performed; $C T$, computed tomography; $L O F$, length of follow-up; $L O S$, length of stay; $M x$, management; $N / A$, not applicable; $N A R$, negative appendicectomy rate; USS, ultrasound scan; $W C C$, white cell count

*Values showing statistical significance

${ }^{\dagger}$ Values demonstrate medians and range

${ }^{\wedge}$ Values demonstrate means 


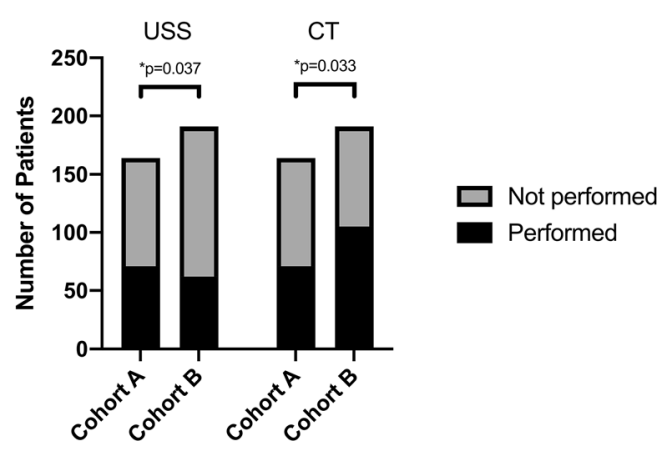

Fig. 1 Change in imaging before and after change in Intercollegiate guidelines. Graph demonstrating distribution of patients who did (black) and did not (grey) undergo abdominal USS and abdominal CT for the investigation of suspected appendicitis in cohort A and cohort B. *Value showing statistical significance using Fisher's exact test. CT computed tomography; USS ultrasound scan

drains and two were readmitted with on-going symptoms and had an open appendicectomy. Within cohort A, an 80-yearold male who underwent laparoscopic appendicectomy for CT-proven appendicitis represented 11 days after discharge with respiratory failure. They were then confirmed to have COVID-19 (SARS-CoV-2 PCR-positive) and died $24 \mathrm{~h}$ later.

The 30-day readmission rate between the two cohorts was comparable (4/161 vs 7/191; $p=0.556)$. Only one patient who was treated non-operatively following introduction of the
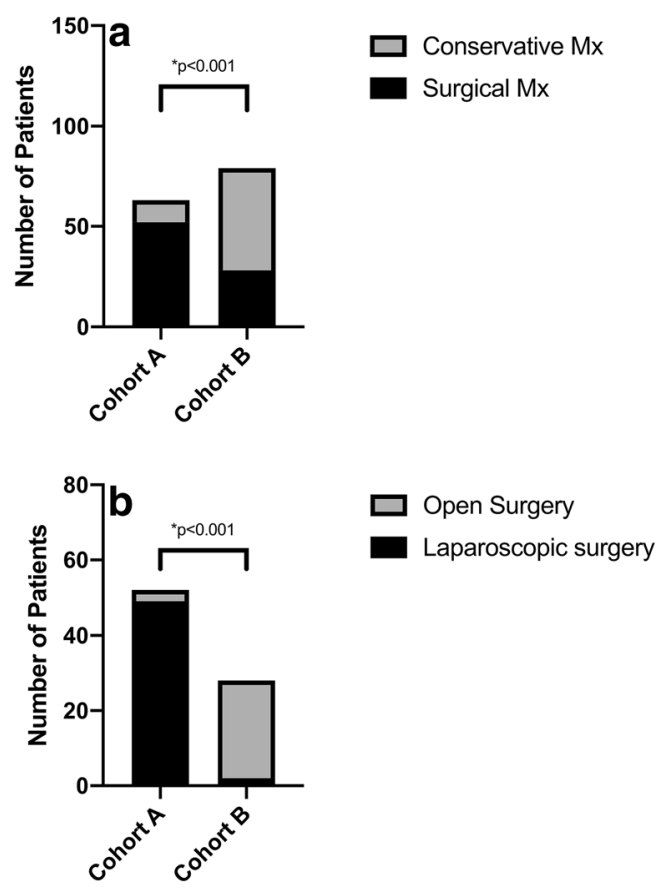

Fig. 2 Management of patients diagnosed with appendicitis before and after change in Intercollegiate guidelines. a Graphs demonstrating number of patients undergoing surgical (black) and non-operative (grey) management in cohorts A and B. b Of patients undergoing surgical management for appendicitis, those managed with a laparoscopic (black) and open (grey) approach in cohorts A and B. *Value showing statistical significance using Fisher's exact test. Mx management guidelines represented and underwent surgery with histology demonstrating a mucinous appendicular tumour.

\section{SARS-CoV-2 PCR testing}

There was a significant increase in SARS-CoV-2 PCR testing (9/164 vs 71/191; $p<0.001)$ (Fig. 5). In cohort A, one patient tested positive. This patient had CT-proven appendicitis and had a laparoscopic appendicectomy. In cohort B, one patient tested positive; this patient was diagnosed with a urinary tract infection.

Two patients from cohort A and three from cohort B had clinically suspected COVID-19 infection based on clinical findings or CT thorax despite negative PCR. Of these five patients, three (one form cohort A and two from B) had appendicitis (CT-proven). In cohort A, this patient was treated non-operatively; they did not develop any complications but were not followed up. In cohort B, both patients were treated non-operatively, neither developed any complications and both were followed up.

\section{Discussion}

This study demonstrates significant changes in the management of appendicitis during the COVID-19 pandemic in the UK. Following introduction of the initial Intercollegiate guidelines patients were more commonly managed non-operatively, spending fewer days in hospital without increase in complication or readmission rate. When surgery was undertaken, laparoscopy was avoided.

Appendicitis is one of the most common surgical emergencies worldwide, and surgery is the mainstay therapy [17]. It is understandable that the modern-day surgeon remains cautious when managing appendicitis without surgical intervention. Our present analysis demonstrates a shift to non-operative management with open surgery preferred over laparoscopy when surgery is required. In cohort A, 52/63 (83\%) patients were managed surgically compared to $28 / 79$ (35\%) in cohort B. Only two patients underwent laparoscopy following introduction of the guidelines compared with 49 previously.

Despite the shift in practice, LOS for patients with appendicitis is shorter following the new guidance and shorter still in those managed non-operatively. In cohort B, patients who underwent surgical management had higher inflammatory markers on admission but without evidence of more CTproven complications. Blood test results appear to influence clinician decision-making more than imaging findings although their clinical value in predicting complicated appendicitis has not been previously demonstrated [18]. Reassuringly, complication rates have remained comparable, demonstrating similar results to a recently published meta-analysis comparing non-operative and operative intervention for appendicitis 


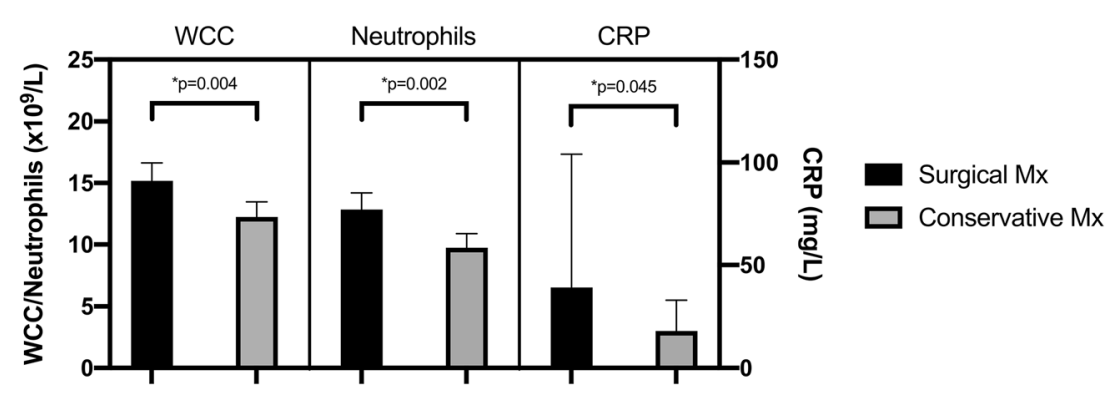

Fig. 3 Differences in inflammatory markers on admission in patients diagnosed with appendicitis after change in Intercollegiate guidelines. Differences in WCC, neutrophil count and CRP on admission following new guideline implementation in patients who went on to

[19]. These results encouragingly show that it may be safe to manage the majority of patients non-operatively without increased short-term complications and a shorter LOS. This is beneficial during a pandemic by reducing the volume of hospital inpatients and reducing stress on operating theatres and staff.

With the rapid spread of the pandemic, the severity of the illness and public health reforms, it is foreseeable that societal anxieties for infection and contact with healthcare workers exist [20]. This may partly explain the apparent reduction in activity in healthcare departments managing non-COVID-19 related disease despite their emergency nature including primary percutaneous cardiac catheterisation [21] and stroke incidence [22]. It is possible that people with milder symptoms or different disease patterns do not present, or present later in their disease course. In the present study, we have not been able to demonstrate a differential proportion of appendicitis diagnoses or NSAP across the two cohorts. Similarly, we did not observe any difference in complications of appendicitis identified with $\mathrm{CT}$ or higher inflammatory markers on presentation.

The suggested increased risk of perioperative viral transmission (to patients and staff) alongside increased morbidity and mortality rates during the pandemic $[23,24]$ has led to the

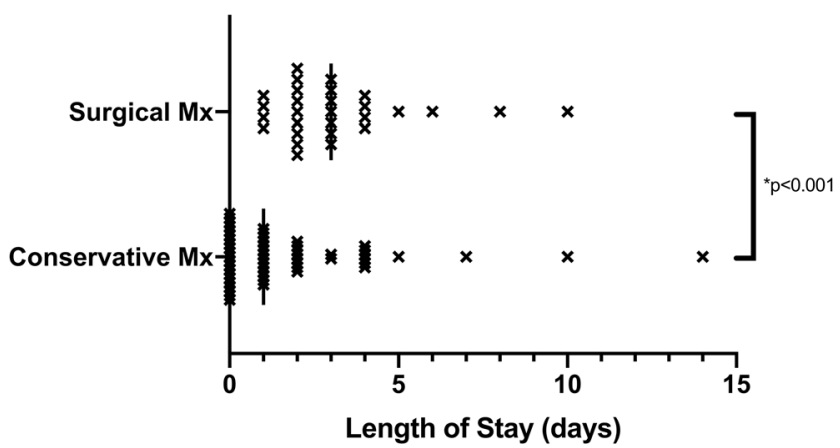

Fig. 4 Length of stay in patients presenting after change in Intercollegiate guidelines. Length of stay in patients managed surgically or nonoperatively for appendicitis presenting after implementation of new guidance. Vertical lines demonstrate median LOS. Vertical line demonstrates median. *Value showing statistical significance using two-tailed unpaired $t$ test. Mx management have surgical (black) and non-operative (grey) management. Error bars demonstrate $95 \%$ confidence intervals. *Value showing statistical significance. CRP C-reactive protein; WCC white cell count

preliminary Intercollegiate guidance. Pre-operative CT thoracic imaging was performed more often following the new guidance although not in all patients undergoing abdominal imaging demonstrating incomplete guidance uptake. PCR testing was performed in only 16 of 28 patients who underwent surgery in cohort B. Limitations in PCR testing availability and time to result may have limited PCR use in all cases, which with increased testing capacity nationally and more rapid testing available may improve. The risk of surgery in those infected or still incubating with SARS-CoV-2 must not be undervalued [24] and is demonstrated with our study's single mortality. This patient presented with abdominal pain and no signs or symptoms of COVID-19. They underwent laparoscopic appendicectomy for CT-proven appendicitis, which on histology was gangrenous. Seven days after discharge, they were readmitted with severe COVID-19 symptoms and acute kidney injury; they died $24 \mathrm{~h}$ later. Such a case demonstrates the surgical dilemma faced by surgeons even in patients who do not have COVID-19 symptoms.

The use of pre-operative imaging in appendicitis, and particularly CT, has been shown to be low in the UK compared to other European countries [25]. It has been suggested that less pre-operative imaging may be responsible for the high NAR in the UK [25]. In the present study, we demonstrate a significant reduction in the use of USS with an increased use of CT. We also demonstrate a lower NAR since the introduction of

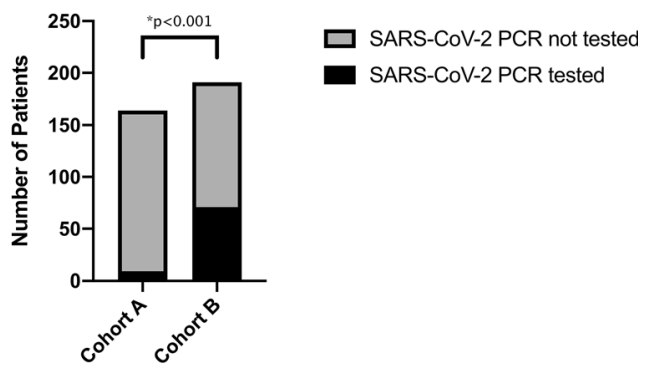

Fig. 5 SARS-CoV-2 PCR testing in patients presenting before and after change in Intercollegiate guidelines. Graph demonstrating distribution of patients who did (black) and did not (grey) undergo SARS-CoV-2 PCR testing in cohort A and B. *Values showing statistical significance using Fisher's exact test. PCR polymerase chain reaction; SARS-CoV-2 severe acute respiratory syndrome coronavirus 2 
the Intercollegiate guidelines. The morbidity associated with surgical removal of a histologically normal appendix is not insignificant (e.g. wound infection, pneumonia or collection), and is reported as $11.9 \%$ [26]. Given the low NAR amongst those operated since the release of the initial Intercollegiate guidelines alongside the increased use of abdominal CT, we postulate that this increased use of cross-sectional imaging improves decision-making. This has been previously reported by the RIFT study group who demonstrated that CT imaging provides excellent discrimination for appendicitis compared with ultrasound [25]. However, the radiation used in CT scanning is not without risk $[27,28]$ especially in young patients. Clinicians therefore have to balance the radiation-related risks with those of negative appendicectomy. Additionally, during the pandemic, there has been a redistribution of junior staff to other departments with concurrent earlier senior review. Senior clinicians may feel more comfortable discharging patients with signs of other pathology or organising early crosssectional imaging.

Long-term recurrence rates following initial non-operative management may be as high as $84 \%$ in 1 year [14] with evidence of persistent recurrence risk for at least 5 years [13]. The surgeon must make a risk-benefit decision, and although recurrence may be high, the risks of surgery during the pandemic alongside limitations in theatre space and staffing due to redeployment may influence management. Interval appendicectomy, once global health has become more favourable, remains a point for consideration and may circumvent recurrence of acute appendicitis or even missed appendicular neoplasia. Surgical teams should follow up patients who are managed non-operatively; within our institution (BHRUT), this was possible via our "hot clinic" which is still operational during the pandemic. Equally, patients should be counselled fully regarding the potential risks of non-operative management, namely the recurrence risk, development of complications or missed pathology.

Our results suggest that non-operative management strategies are safe for patients with acute appendicitis, a finding supported by the wider literature [12, 29]. However, the optimal non-operative treatment strategy for acute appendicitis remains uncertain. The recently published World Society of Emergency Surgery guidelines recommend that patients receive intravenous antibiotic therapy initially, before a subsequent switch to oral antibiotics [9]. There is however evidence to suggest that supportive care alone without antibiotics is a safe alternative [30]. We await the results of the APPAC II [31] and III [32] trials, which we hope will provide an optimal evidence-based non-operative strategy.

\section{Limitations}

The scope of our current analysis was to present early data on the change in diagnosis, management and outcomes of appendicitis during the COVID-19 pandemic to guide current management as the health crisis evolves. Due to the immediate need for evidence on the management of appendicitis in adults during the COVID-19 pandemic, we have a limited data set and only completed 30 days of follow-up. These factors also increase our likelihood of type 2 errors. Longer-term outcomes for non-operatively managed patients including operative rate, recurrence or other complications [13] will be needed which we plan to report in the future. Patient compliance with outpatient follow-up and outpatient department capacity may be a limiting factor when managing patients nonoperatively. The role of virtual clinics and telemedicine may circumvent this.

\section{Conclusions}

Management of adults presenting with acute appendicitis in the UK is significantly different since the release of the initial Intercollegiate general surgery guidance. More patients are being treated non-operatively; they are staying fewer days in hospitals and have no increased risk of short-term complications or readmission. More patients are undergoing crosssectional imaging which may partly explain a reduced NAR. Guidelines are being followed with regard to the use laparoscopy with most patients having open surgery if required. Further updates to the intercollegiate guidance will likely further change practice.

The long-term complications of increased use of nonoperative management are not clear. Further studies reporting long-term complications of this change in management will be required.

Supplementary Information The online version contains supplementary material available at https://doi.org/10.1007/s00423-020-02023-6.

Authors' contributions WE and NHB contributed equally as first authors. WE and NHB contributed to conception and research design. NHB, CS, $\mathrm{ED}, \mathrm{SM}, \mathrm{WM}, \mathrm{MC}$ and JE performed data collection for their respective centres. WE, NHB and CS performed data analysis and interpretation and drafted the manuscript. WE, NHB, MM and VS critically revised the manuscript. All authors gave final approval of the manuscript for publication.

Data availability Data available for 10 years on request.

\section{Compliance with ethical standards}

Conflict of interest The authors declare that they have no conflict of interest.

Ethics approval The UK National Research Ethics Service decision tool (http://www.hra-decisiontools.org.uk) confirmed this study does not require ethical approval. It was registered within each trust as a service evaluation. 
Consent to participate Not applicable.

Consent for publication Approved by all authors.

Code availability Not applicable.

Abbreviations CI, 95\% confidence interval; COVID-19, coronavirus disease 2019; CRP, C-reactive protein; CT, computed tomography; EHR, electronic health record; LOF, length of follow-up; LOS, length of stay; Mx, management; N/A, not applicable; NAR, negative appendicectomy rate; NSAP, non-specific abdominal pain; PCR, polymerase chain reaction; SARS-CoV-2, severe acute respiratory syndrome coronavirus 2; USS, ultrasound scan; WCC, white cell count

\section{References}

1. COVIDSurg Collaborative, Nepogodiev D, Bhangu A (2020) Elective surgery cancellations due to the COVID-19 pandemic: global predictive modelling to inform surgical recovery plans. $\mathrm{Br}$ J Surg. https://doi.org/10.1002/bjs.11746

2. Søreide K, Hallet J, Matthews JB, Schnitzbauer AA, Line PD, Lai PBS, Otero J, Callegaro D, Warner SG, Baxter NN, Teh CSC, NgKamstra J, Meara JG, Hagander L, Lorenzon L (2020) Immediate and long-term impact of the COVID-19 pandemic on delivery of surgical services. Br J Surg. https://doi.org/10.1002/bjs.11670

3. COVIDSurg Collaborative (2020) Mortality and pulmonary complications in patients undergoing surgery with perioperative SARSCoV-2 infection: an international cohort study. Lancet. 396:27-38. https://doi.org/10.1016/S0140-6736(20)31182-X

4. Gov.UK Coronavirus (COVID-19) in the UK. https://coronavirus. data.gov.uk/. Accessed 10 Jun 2020

5. Royal College of surgeons of England updated Intercollegiate general surgery guidance on COVID-19. In: Royal College of Surgeons. https://www.acpgbi.org.uk/content/uploads/2020/03/ Updated-Intercollegiate-General-Surgery-Guidance-on-COVID19-final-with-logos13.pdf. Accessed 7 Oct 2020

6. Mowbray NG, Ansell J, Horwood J, Cornish J, Rizkallah P, Parker A, Wall P, Spinelli A, Torkington J (2020) Safe management of surgical smoke in the age of COVID-19. Br J Surg. https://doi.org/ 10.1002/bjs.11679

7. Zheng MH, Boni L, Fingerhut A (2020) Minimally invasive surgery and the novel coronavirus outbreak: lessons learned in China and Italy. Ann Surg 272:e5-e6. https://doi.org/10.1097/SLA. 000000000003924

8. Stewart B, Khanduri P, McCord C, Ohene-Yeboah M, Uranues S, Vega Rivera F, Mock C (2014) Global disease burden of conditions requiring emergency surgery. Br J Surg 101:e9-e22. https://doi.org/ 10.1002/bjs. 9329

9. Di Saverio S, Podda M, De Simone B et al (2020) Diagnosis and treatment of acute appendicitis: 2020 update of the WSES Jerusalem guidelines. World J Emerg Surg 15:27. https://doi.org/ 10.1186/s13017-020-00306-3

10. Jaschinski T, Mosch CG, Eikermann M, Neugebauer EAM, Sauerland S, Cochrane Colorectal Cancer Group (2018) Laparoscopic versus open surgery for suspected appendicitis. Cochrane Database Syst Rev 11:CD001546. https://doi.org/10. 1002/14651858.CD001546.pub4

11. Alnaser MKH, Hassan QA, Hindosh LN (2018) Effectiveness of conservative management of uncomplicated acute appendicitis: a single hospital based prospective study. Int Surg J Open 10:1-4. https://doi.org/10.1016/j.ijso.2017.11.007

12. Podda M, Gerardi C, Cillara N, Fearnhead N, Gomes CA, Birindelli A, Mulliri A, Davies RJ, di Saverio S (2019) Antibiotic treatment and appendectomy for uncomplicated acute appendicitis in adults and children: a systematic review and meta-analysis. Ann Surg 270: 1028-1040. https://doi.org/10.1097/SLA.0000000000003225

13. Salminen P, Tuominen R, Paajanen H, Rautio T, Nordström P, Aarnio M, Rantanen T, Hurme S, Mecklin JP, Sand J, Virtanen J, Jartti A, Grönroos JM (2018) Five-year follow-up of antibiotic therapy for uncomplicated acute appendicitis in the APPAC randomized clinical trial. JAMA 320:1259-1265. https://doi.org/10. 1001/jama.2018.13201

14. Collard M, Lakkis Z, Loriau J, Mege D, Sabbagh C, Lefevre JH, Maggiori L (2020) Antibiotics alone as an alternative to appendectomy for uncomplicated acute appendicitis in adults: changes in treatment modalities related to the COVID-19 health crisis. J Visc Surg 157:S33-S42. https://doi.org/10.1016/j.jviscsurg.2020.04. 014

15. English W, Habib Bedwani N, Smith C, Shatkar V (2020) Investigation and management of suspected appendicitis during the COVID-19 pandemic. Br J Surg 107:e337-e338. https://doi. org/10.1002/bjs. 11787

16. Dindo D, Demartines N, Clavien P-A (2004) Classification of surgical complications: a new proposal with evaluation in a cohort of 6336 patients and results of a survey. Ann Surg 240:205-213

17. Addiss DG, Shaffer N, Fowler BS, Tauxe RV (1990) The epidemiology of appendicitis and appendectomy in the United States. Am J Epidemiol 132:910-925. https://doi.org/10.1093/oxfordjournals. aje.a115734

18. Al-gaithy ZK (2012) Clinical value of total white blood cells and neutrophil counts in patients with suspected appendicitis: retrospective study. World J Emerg Surg 7:32. https://doi.org/10.1186/17497922-7-32

19. Yang Z, Sun F, Ai S, Wang J, Guan W, Liu S (2019) Meta-analysis of studies comparing conservative treatment with antibiotics and appendectomy for acute appendicitis in the adult. BMC Surg 19. https://doi.org/10.1186/s12893-019-0578-5

20. Torales J, O'Higgins M, Castaldelli-Maia JM, Ventriglio A (2020) The outbreak of COVID-19 coronavirus and its impact on global mental health. Int J Soc Psychiatry 66:317-320. https://doi.org/10. 1177/0020764020915212

21. Garcia S, Albaghdadi MS, Meraj PM, Schmidt C, Garberich R, Jaffer FA, Dixon S, Rade JJ, Tannenbaum M, Chambers J, Huang PP, Henry TD (2020) Reduction in ST-segment elevation cardiac catheterization laboratory activations in the United States during COVID-19 pandemic. J Am Coll Cardiol 75:2871-2872. https://doi.org/10.1016/j.jacc.2020.04.011

22. Siegler JE, Heslin ME, Thau L, Smith A, Jovin TG (2020) Falling stroke rates during COVID-19 pandemic at a comprehensive stroke center: cover title: falling stroke rates during COVID-19. J Stroke Cerebrovasc Dis 29:104953. https://doi.org/10.1016/j. jstrokecerebrovasdis.2020.104953

23. Myles PS, Maswime S (2020) Mitigating the risks of surgery during the COVID-19 pandemic. Lancet 396:2-3. https://doi.org/10.1016/ S0140-6736(20)31256-3

24. Lei S, Jiang F, Su W, Chen C, Chen J, Mei W, Zhan LY, Jia Y, Zhang L, Liu D, Xia ZY, Xia Z (2020) Clinical characteristics and outcomes of patients undergoing surgeries during the incubation period of COVID-19 infection. EClinicalMedicine 21:100331. https://doi.org/10.1016/j.eclinm.2020.100331

25. Bhangu A, RIFT Study Group on behalf of the West Midlands Research Collaborative (2020) Evaluation of appendicitis risk prediction models in adults with suspected appendicitis. Br J Surg 107: 73-86. https://doi.org/10.1002/bjs.11440

26. Lee M, Paavana T, Mazari F, Wilson T (2014) The morbidity of negative appendicectomy. Ann R Coll Surg Engl 96:517-520. https://doi.org/10.1308/003588414X13946184903801

27. Lahaye MJ, Lambregts DMJ, Mutsaers E, Essers BAB, Breukink S, Cappendijk VC, Beets GL, Beets-Tan RGH (2015) Mandatory 
imaging cuts costs and reduces the rate of unnecessary surgeries in the diagnostic work-up of patients suspected of having appendicitis. Eur Radiol 25:1464-1470. https://doi.org/10.1007/s00330-0143531-0

28. Rogers W, Hoffman J, Noori N (2015) Harms of CT scanning prior to surgery for suspected appendicitis. Evid Based Med 20:3-4. https://doi.org/10.1136/ebmed-2014-110075

29. Sallinen V, Akl EA, You JJ, Agarwal A, Shoucair S, Vandvik PO, Agoritsas T, Heels-Ansdell D, Guyatt GH, Tikkinen KAO (2016) Meta-analysis of antibiotics versus appendicectomy for nonperforated acute appendicitis. Br J Surg 103:656-667. https://doi. org/10.1002/bjs. 10147

30. Park HC, Kim MJ, Lee BH (2017) Randomized clinical trial of antibiotic therapy for uncomplicated appendicitis. Br J Surg 104: 1785-1790. https://doi.org/10.1002/bjs.10660
31. Haijanen J, Sippola S, Grönroos J et al (2018) Optimising the antibiotic treatment of uncomplicated acute appendicitis: a protocol for a multicentre randomised clinical trial (APPAC II trial). BMC Surg 18:117. https://doi.org/10.1186/s12893-018-0451-y

32. Sippola S, Grönroos J, Sallinen V, Rautio T, Nordström P, Rantanen T, Hurme S, Leppäniemi A, Meriläinen S, Laukkarinen J, Savolainen H, Virtanen J, Salminen P (2018) A randomised placebo-controlled double-blind multicentre trial comparing antibiotic therapy with placebo in the treatment of uncomplicated acute appendicitis: APPAC III trial study protocol. BMJ Open 8: e023623. https://doi.org/10.1136/bmjopen-2018-023623

Publisher's note Springer Nature remains neutral with regard to jurisdictional claims in published maps and institutional affiliations. 